\title{
IL NOVECENTISMO: "UN'IMPRESA CONTRO IL DIAVOLO«. NOTE SUL PROGETTO CULTURALE ED ESTETICO BONTEMPELLIANO
}

\section{LINEE DI UN RINNOVAMENTO ARTISTICO-CULTURALE}

Massimo Bontempelli fu capace di rimettersi più volte in gioco nella sua vita rivedendo le proprie posizioni in fatto di scrittura e critica culturale oltre che di politica ${ }^{1}$. Qui ci si soffermerà sulle linee socioculturali e poetologiche che improntano la sua attività tra la metà degli anni Venti e la fine degli anni Trenta ovvero nella fase in cui lavorò all'elaborazione e difesa di quella tendenza di rinnovamento culturale che fu il Novecentismo. L'intento è di evidenziare le caratteristiche salienti delle posizioni abbracciate in quegli anni, compresi i nodi che vi emergono. Lo faremo sulla scorta de L'avventura novecentista (1938), la silloge di scritti teorici, critici e polemici in cui lo scrittore raccolse articoli, lettere e note varie, fra cui gli articoli programmatici sul Novecentismo che aveva pubblicato sui Cahiers du "900" (1926-1929) riproponendoli in traduzione italiana assieme ad alcuni interventi di ragguaglio e commento ${ }^{2}$. Nel loro insieme quei testi, ordinati secondo un criterio tematico, permettono di seguire sia i presupposti in cui si concretizzò la teorizzazione del Novecentismo, sia la difesa di tali posizioni negli anni successivi all'esperienza della rivista mettendo così in luce il disegno di fondo della riflessione estetica e culturale di questo intellettuale in uno dei periodi più decisivi della sua attività ${ }^{3}$.

Il discorso affrontato ne L'avventura novecentista è ora polemico, ora analitico, ora programmatico e, sebbene intenda avere una portata generale, nasce dal bilancio dell'esperienza estetica dell'autore delineandone a posteriori anche la personale poetica ${ }^{4}$.

\footnotetext{
1 A lungo Bontempelli credette nella capacità innovativa del fascismo, come testimoniano la sua adesione al partito e la difesa d'iniziative culturali fasciste. Ciò non gli precluse, tuttavia, ancora prima di passare all'opposizione, di esprimere giudizi critici e assumere posizioni di rifiuto verso determinate richieste (in primo luogo di propaganda) avanzate da funzionari politici. Rende conto di quella svolta anche il volumetto di scritti critici Dignità dell'uomo (Bontempelli, 1946).

2 La rivista era stata fondata nel 1926 assieme a Malaparte e i primi cinque numeri uscirono in francese. Già la sola scelta di lingua indicava l'intenzione dei redattori di aprirvi un dibattito letterario di respiro europeo.

3 A segnalare quanto le tesi sostenute negli interventi saggistici degli anni Venti e Trenta stiano in collegamento con posizioni difese già prima della guerra, è l'inserzione nel volume, quale lettera prefatoria, di uno scritto del 1914 in cui si lamenta lo stato di stagnazione della letteratura contemporanea.

4 La presenza di figure di candidi in diverse opere stilate prima del '26 mostra che una prospettiva capace di stupore e meraviglia sulle cose fu centrale della poetica bontempelliana ancor prima della formulazione teorica del realismo magico.
} 
Dietro la voce plurale che talvolta affiora in quelle pagine, ad esempio negli articoli già usciti sui Cahiers, a delinearsi è la prospettiva di un io, e precisamente di un io sicuro di restituire con le proprie osservazioni e i propri giudizi di valore un'immagine centrata dei problemi della cultura italiana del tempo. Bontempelli nomina con reticenza perfino i collaboratori ai Cahiers, si pensi ad esempio a Nino Frank, che fu la sua spalla forte nel lavoro di preparazione e lancio della rivista ${ }^{5}$, ed è anche poco disponibile a riconoscere i meriti di altri intellettuali contemporanei impegnati a sviluppare esperienze artistiche innovative; vi fanno eccezione pochi, fra cui Pirandello, Savinio, De Chirico.

Quanto alle scelte discorsive, non può sfuggire il tono esortativo talvolta presente negli scritti del volume, il che evidenzia quanto il loro autore recuperasse suo malgrado, anche a livello retorico, elementi dell'esperienza avanguardista e nella fattispecie modalità espressive del genere del manifesto ${ }^{6}$. Ma il linguaggio adottato tiene conto anche della scrittura giornalistica; ciò non è casuale considerando il favore che Bontempelli le accordava, al punto da ritenere che questa avrebbe avuto molto da insegnare anche ai narratori. Attraverso un registro linguistico colloquiale ed espressioni incisive, prive di smussature, spesso addirittura irriverenti e sempre orientate a far presa sul lettore, in quegli scritti egli combatte, da una parte, una battaglia per lo svecchiamento della cultura italiana e difende, dall'altra, la specificità dell'ambito letterario. Un ambito che concepisce - con precisa sensibilità novecentesca - nella sua piena autonomia, ma senza che ciò implichi la tesi di una separatezza della realtà estetica da quella storica e sociale. Tutt'altro. Nel volume s'individua, infatti, una viva attenzione anche per precisi aspetti socioculturali così che le due prospettive, este-

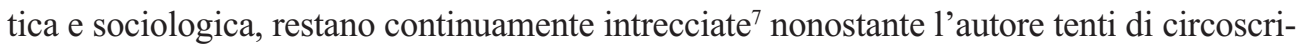
vere le riflessioni su fatti di costume in un capitolo specifico, dedicato a interventi polemici su alcuni mali tipici della società italiana. In quel capitolo denuncia, tra altri aspetti, la tendenza degli italiani a fare del poverismo una virtù, il loro abbarbicarsi ad abitudini e codici culturali anacronistici, l'insistenza a valorizzare la lettura di testi sorpassati, fra cui quella »immoralissima« di Cuore (De Amicis, 1886), ${ }^{8}$ e polemizza di conseguenza sull'esistenza di istituzioni - famiglia, scuola e università - diseducanti. Scriveva ad esempio: »[q]uanto alla famiglia, in generale il giovane universitario esce da famiglie borghesi, la cui cultura è fatta tutta sulla Domenica del Corriere e sulla Settimana enigmistica. ${ }^{9}$

Il solo fatto che Bontempelli, anticipando posizioni che sarebbero circolate in diverse teorie letterarie del XX secolo, assumesse l'opera d'arte come una forma comunicativa

5 La centralità di Frank nell'impresa dei Cahiers è evidenziata nel carteggio Alvaro, Bontempelli, Frank, 1995.

6 Lo ricorda anche Sandra Milanko nella sua recente monografia »È indubbio che questa arte di fare i manifesti è stata una grande lezione per tutti i seguaci o non del fondatore del futurismo, non solo nei termini stilistici, ma anche nella stessa scelta dei termini o immagini di stampo futurista che sarebbero stati adottati per esempio da un Papini o riciclati negli anni Venti dallo stesso Bontempelli [...].« Milanko, 2014, 13.

7 Cfr. AN, 50-52.

8 Cfr. AN, 151.

9 AN, 156. 
e v'individuasse una portata politica, indica il nesso sussistente, nel suo orizzonte di pensiero, fra arte e società, fra opera e ricettori. Osservava al proposito: » [L]'arte è comunicazione, è linguaggio. Perciò essa presuppone l'uomo consociato. Perciò l'arte ha sempre una portata politica. Un'opera d'arte, se ha valore artistico, ha implicitamente anche valore politico. $\ll^{10}$

I fini che guidano le due battaglie appena segnalate, difendere cioè la specificità e autonomia dell'arte e della letteratura da istanze poetologicamente normative e da pressioni di potere, e tenere conto allo stesso tempo del contesto socioculturale in cui si realizzano, in particolare quello di una realtà di massa, possono forse apparire poco conciliabili fra loro; trovano tuttavia plausibilità se si considerano i presupposti del progetto utopico di rinnovamento culturale in cui s'inseriscono, che è la lettura proposta da Buonanno in un convincente articolo di qualche anno $\mathrm{fa}^{11}$. Di quelle frizioni, o piuttosto sfide, daremo nel seguito qualche esempio.

La necessità di un rinnovamento culturale è in effetti la parola d'ordine di Bontempelli. La sua spina nell'occhio era un mondo intellettuale che vedeva arroccato su dei valori e delle concezioni estetiche sorpassate oltre che cieco di fronte al nuovo pubblico. Oggetto di rifiuto era un'arte giudicata infeconda, tardoromantica e tardoverista ${ }^{12}$. A contrastarla avrebbe dovuto essere un'attività artistica e letteraria intesa come rischio, da affrontarsi con senso di avventura ${ }^{13}$ in termini antitetici a quelli cari allo spirito borghese $^{14}$. Di fronte all'opera d'arte, scriveva, bisognerebbe: $»[\mathrm{t}]$ emere sempre che non si tratti d'ispirazione, ma di trucco. Tanti saluti ai bei comodi del realismo, alle truffe dell'impressionismo. ${ }^{15} \mathrm{La}$ sua arringa contro residui romantici nell'orizzonte culturale italiano, e nella fattispecie in quello letterario, andava a colpire non solo l'immagine romantica dello scrittore come genio e della scrittura come ispirazione, ma implicitamente anche la corrente dell'espressionismo e del frammentismo lirico, rappresentata in Italia soprattutto dai vociani. Questi si erano impegnati per un costruttivo rinnovamento della critica e della letteratura, ma da una prospettiva che lasciava uno spazio centrale a quella soggettività che Bontempelli invece combatteva ${ }^{16}$. Non mancano, in tale polemica contro una cultura inadeguata al nuovo secolo, delle note provocatorie secondo cui gli autori avrebbero dovuto affidarsi ai consigli dei librai. Il che indica non solo un interesse di questo intellettuale per il rapporto fra letteratura e mercato, ma anche una concezione

\footnotetext{
$10 \quad$ AN, 314.

11 Cfr. Buonanno, 2003. Su certi concetti problematici presenti nella riflessione teorica bontempelliana, in particolare quello di mito, si veda anche l'istruttivo articolo di Airoldi Namer (1975). Per un bilancio storico del Novecentismo resta ancora utile Falqui $(1953,73-130)$.

12 Cfr. la lettera prefatoria e gli interventi »Avanzi di una polemica«, AN, 264-265.

13 Cfr. AN, 36.

14 Cfr. AN, 136.

15 AN, 19.

16 Per la nuova impronta che La Voce ebbe a partire dal dicembre del '14 sotto la direzione di De Robertis, cfr. Falqui, 1967.
} 
dell'attività letteraria come lavoro, ben lontana da quella difesa, tra l'altro, dall'estetica crociana. »Bisogna ricordarci« scriveva »che la materia prima della poesia è il mestiere: l'arte è un mestiere mediante il quale si raggiunge qualche volta la poesia [...].«17

Pensate come inadeguate ai tempi, e fatte oggetto di critica e d'ironia, erano tanto le posizioni dei futuristi, quanto quelle di letterati del primo Novecento non avanguardisti e propugnatori di un concetto puro dell'arte e del linguaggio. Era in altri termini una distanza dalle posizioni degli intellettuali che Brioschi ne la Critica della ragion poetica avrebbe indicato rispettivamente come difensori del criterio della »Purezza« e difensori del criterio del $»$ Nuovo« (o con più esattezza del nuovo per il nuovo) ${ }^{18}$. Dei primi, individuabili nei rondisti, Bontempelli rifiuta la difesa di una lingua pura ${ }^{19}$ e l'impermeabilità verso il grande pubblico ${ }^{20}$, dei futuristi non lo convince la retorica, il soggettivismo e il persistere nell'imporre regole formali di scrittura. Riconosce tuttavia la positiva funzione di »sgomberamento« che ebbero nell' ambiente culturale italiano del primo Novecento ${ }^{21}$.

La capacità di considerare senza pregiudizi la produzione artistica per il largo consumo e la rivalutazione delle arti applicate lo avvicinano tuttavia ai futuristi anche nella sua fase novecentista e più di quanto egli fosse stato disposto ad ammettere in quegli anni. Dietro alla comune difesa di svecchiamento culturale, le modalità e gli strumenti d'intervento individuati erano comunque ben diversi: non si trattava, tra l'altro, di far piazza pulita del passato, ma al contrario di rivalutare la grande lezione dei classici.

Mosso da un'esigenza costruttiva di rinnovamento che trapela anche nella ricerca d'immagini e di un lessico tratti dall'architettura ${ }^{22}$, egli traccia dunque nei Cahiers, tra il 1926 e il 1929, le linee di un progetto per l'arte e la letteratura che avrebbe voluto essere adeguato ai tempi. E su quel progetto sarebbe tornato anche in seguito con delle precisazioni e integrazioni di cui rendono conto appunto diversi articoli de L'avventura novecentista. Dall'arte pretendeva innanzitutto trasfigurazione ${ }^{23}$, capacità comunicativa, anonimità, autonomia rispetto al terreno di crescita. Tali caratteristiche non indicavano, però, solo il profilo richiesto al prodotto artistico per il nuovo secolo: erano pensate pure in termini sovraepocali come peculiari dei classici. E allora, in una dinamica a doppio

\footnotetext{
17 AN, 74, corsivo nel testo.

18 Cfr. Brioschi, 2002, 31.

19 »Ho una gran diffidenza verso l'aggettivo "puro", di cui i soccorritori fanno un grande abuso, e che non vuol proprio dir niente.« AN, 50.

20 In un articolo degli anni Trenta afferma: »[...] oggi il pericolo non è nella "letteratura", ma nel suo contrario. [...]. La protezione politica favorisce involontariamente il più mediocre dilettantismo, e la schiera degli orecchianti e dei faciloni se ne ingrossa.« AN, 299.

21 Cfr. AN, 35-40, versione it. dell'articolo »Analogies« apparso sul nr. IV dei Cahiers.

22 Vi ha già richiamato l'attenzione Piscopo (2001, 207-214) osservando come Bontempelli non fosse del resto solo nella difesa del »canone dell'architettonicità

23 Bontempelli è ben attento a non confondere »trasfigurazione« con »deformazione«. In una nota su De Chirico scriveva: »[...] verità suprema dell'arte [...] è la trasfigurazione (non la «deformazione» come molti s'ostinano a dire).« Bontempelli, 2006, 22. La nota compare in una recente antologia a cura di E. Pontiggia di scritti sul realimo magico comprendente alcuni articoli dei Cahiers e altri brevi interventi bontempelliano di estetica.
} 
senso, l'opera novecentista assurgeva al piano dei classici e i classici a loro volta erano misurati col metro del Novecentismo. Bontempelli non si stancava di ripetere che l'Odissea aveva avuto la capacità di parlare a tutti ${ }^{24}$.

Nella sua riflessione, gli autori di opere che tennero nel tempo erano state in grado di dire l'universale attraverso il fatto singolo, di svincolarsi dal contingente proiettandosi verso il nuovo. Si precisa così anche il modo in cui egli intendeva sia la storia letteraria (lo sviluppo della quale poggiava a suo avviso su dinamiche interne, sulla capacità dei grandi autori di assorbire quanto tramandato per andar oltre), sia la tradizione, il cui motore funzionerebbe, a seguire il suo ragionamento, grazie al connubio di continuità, innovazione, indipendenza: »Perché la tradizione c'è, sì, non è vero che sia una invenzione: ma è fatta della continuità intima, profonda, tra manifestazioni di inaspettata novità. Ognuno degli autori che la tradizione accoglie, è un ribelle all'aspetto immediatamente precedente di essa: era uno che della tradizione santissimamente se ne infischiava. ${ }^{25}$ I testi assurti a classici rappresentavano ai suoi occhi i valori del sovratemporale, sovraspaziale, sovra individuale, tutti valori che si augurava potessero imporsi come distintivi di un Novecento da lui ritenuto, alle soglie degli anni '20, non ancora veramente cominciato. È in essi che cercava esempi autorevoli di fondamento alla nuova letteratura ${ }^{26}$.

In una simile concezione estetica emerge allora un idealismo (di origine romantica, più che platonica) ${ }^{27}$, secondo cui l'arte è impossibilitata a mantenere una forma definitiva. »[L]'arte ha per natura fondamentale di non ritrovare mai, di inventare continuamente la forma nuova. ${ }^{28}$ Tale posizione portava Bontempelli a esprimere considerazioni attualissime in quegli anni - e centrali fra l'altro in quell'ambiente formalista ancora del tutto ignoto al mondo culturale occidentale - sull'opportunità di guardare allo sviluppo dell'arte e della letteratura a partire da dinamiche interne di rinnovamento. Nel contesto novecentista la poiesis avrebbe dovuto concepirsi come rischio, come trasgressione rispetto al già consolidato, come continua ricerca di rinnovamento. Tali posizioni non precludevano tuttavia, come già osservato, la difesa di un'arte e di una letteratura popolari, nello specifico di una narrativa capace di contaminarsi con il cinema, con la cronaca e con generi letterari considerati meno alti come il romanzo poliziesco. E qui emerge appunto quello scopo duplice, difficilmente conciliabile, tra l'idea di un'arte capace di aprirsi al nuovo, tesa a evitare percorsi consolidati, straniante, per assumere la categoria proposta da Šklovskij (Iskusstvo kak priëm/L'arte come procedimento, 1917), e la richiesta che fosse popolare. Scopo difficilmente realizzabile perché, come noto, un'arte e una letteratura tese a cercare se stesse, pronte a oltrepassare i risultati raggiunti

\footnotetext{
24 Cfr. AN, 145, 155, 298.

25 AN, 34, corsivo nel testo.

$26 \mathrm{AN}, 39$.

$27 »[\mathrm{~L}]$ 'arte non è se non un riflesso di quella raggiunta attuazione dell'uno col tutto [...].« AN, 320.

28 AN, 331. La perfezione raggiunta appare a Bontempelli come irrigidimento ed esaurimento: $»[\ldots]$ come stile non c'è mai da imparare dai più perfetti, ma se mai al contrario dai più semplici e grossolani e iniziali [...].« $\mathrm{AN}, 59$, corsivo nel testo.
} 
rimettendosi in discussione e sperimentando il nuovo, tendono a tradire le attese del grande pubblico e si rendono facilmente impopolari. Lo coglieva chiaramente Ortega y Gasset all'altezza dello stesso periodo (1925) ne La deshumanización del Arte e Ideas sobre la novela osservando appunto (anche a proposito della pittura) per quali ragioni l'arte contemporanea, con la sua tendenza all'astrattismo, non potesse essere accolta che da élites ${ }^{29}$.

Il realismo magico, che è la poetica o piuttosto (con termine preferito dal suo teorico) la "tendenza" perseguita entro l'utopia culturale del Novecentismo, poggia sulla coscienza che l'arte non può essere mimesi. Compito dello scrittore e dell'artista sarebbe stato elaborare, attraverso l'immaginazione, situazioni tratte dalla realtà fenomenica, per mostrare il doppio fondo di quella realtà ${ }^{30}$. In tal senso l'arte era intesa come una sorta di magia ${ }^{31}$. Era in gioco la difesa di un fare artistico e di una visione del mondo capaci di valorizzare lo stupore esperibile anche in situazioni quotidiane, in momenti capaci di lasciare immaginare una dimensione ulteriore. Era richiesta, con ciò, quell'attitudine a staccarsi dal contingente che Bontempelli riconosceva essere il carattere basilare della poesia ${ }^{32}$. Una simile posizione solleva la questione del rapporto tra letteratura e attualità, questione che egli affrontò tra l'altro in uno scritto di obiezione alle richieste agli scrittori da parte dell'ambiente fascista. Vi sostiene che all'artista spetti rappresentare il proprio tempo in maniera indiretta. »La "rappresentazione del tempo" che più o meno artificiosamente sogliamo trovare nei poeti, è sempre ottenuta in modo indiretto. « E ribadiva ancora: »La "glorificazione" di una condizione storica contemporanea non può essere il tema di un'opera d'arte [...].. $\ll^{33}$ La portata politica e morale di un'opera d'arte, osservava ancora in un altro contesto, è lungi dal trovarsi nell'esplicita trattazione di un tema politico o morale. »Ecco il solito grossolano errore. Il più o meno di moralità d'uno scritto, si crede consista nelle idee esposte, nei costumi raccontati; no: esso sta tutto in quel senso ineffabile che è l'arte, senso di umanità, profondità, naturalità, aura religiosa, che è dato come ho detto dal tono, e che solo costituisce la vera sola efficace portata morale dell'arte. $\aleph^{34}$ L'esigenza di »inaderenza«, di una distanza rispetto al presente (raggiungibile non da ultimo attraverso l'ironia) ${ }^{35} \mathrm{e}$ la necessità di uno sguardo nuovo sul mondo, da primitivi, erano centrali entro il progetto novecentista ${ }^{36}$. Altrettanto basilare era la richiesta che l'opera letteraria lasciasse spazio alla fantasia e all'inverosimile,

29 Ortega y Gasset, 1997.

30 Giustamente Airoldi Namer $(1975,266)$ fa osservare che tale realtà è pensata talvolta come altra, talvolta come intrecciata a quella fenomenica.

31 Nel capitolo »Lettre d'Italie « (1945) Contini ridimensiona la portata che quel concetto assume nell'opera dello scrittore: »[...] la prétendue magie de Bontempelli a son origine dans la trouvaille d'une agudeza, d'une «pointe».« Contini, 1972, 280.

32 Cfr. AN, 304 e 331.

33 AN, 309 e 312.

34 AN, 325-326, corsivo nel testo..

35 "L'ironia è la forma artistica del pudore al cospetto dei nostri sentimenti, è un modo di allontanarci dal contingente, di liberarci da un'aderenza troppo minuta con le superfici delle cose.« AN, 27.

36 In un articolo del '38 intitolato »Primitivi d'un'epoca« si legge: »Quello e non altro era poesia: popolare il mondo di creature immaginate, in cui le esperienze quotidiane si siano trasformate e risolte.« AN, 304. 
s'incentrasse sull' intreccio ${ }^{37} \mathrm{e}$, in analogia ai miti antichi, consistesse di »favole e personaggi $\ll$ che potessero »correre il mondo $\aleph^{38}$ uscendo alleggerita dal peso d'istanze autoriali ${ }^{39}$. Al soggettivismo Bontempelli contrapponeva, infatti, l'anonimità dissuadendo gli scrittori dal trasporre nei propri testi delle esperienze personali. »L'ideale supremo di tutti gli artisti dovrebbe essere: diventare anonimi. $\aleph^{40}$ In un altro contesto scriveva: »[...] il poeta non può rendere se non sentimenti che non prova. ${ }^{41}$ Analogamente rifiutava un tipo di critica mirante a cercare nelle opere i sentimenti dell'autore. Ne rende conto fra l'altro un articolo del 1933: »[v]orrei una critica che dimenticasse nel modo più perfetto l'autore e le sue intenzioni. Che considerasse ogni opera come un oggetto, come valore a sè [SIC] stante e assoluto. $«^{42}$ Ed è una posizione che lo avvicina di nuovo enormemente a quelle difese dai formalisti. In ragione di un rifiuto della soggettività, arrivava perfino a escludere la poesia lirica dal quadro progettuale di una nuova letteratura. Posizione per certi versi poco comprensibile considerando l'ottima tenuta di quel genere e la profonda capacità di rinnovamento che proprio la poesia in Italia aveva mostrato negli anni che precedettero e seguirono la prima guerra mondiale.

Da anticrociano, Bontempelli lottava contro la cesura fra arte pura e applicata, fra arte alta e popolare. Riteneva necessario o l'abbandono di forme artistiche che avevano ormai esaurito la propria funzione o il loro radicale rinnovamento attraverso stimoli rinvenibili anche in generi di consumo. ${ }^{43}$ A partire da tali posizioni intravedeva, ad esempio, nel teatro di varietà e perfino nei grandi incontri sportivi dei possibili sostituti a quel teatro di prosa che a suo giudizio non aveva saputo rinnovarsi perdendo il suo elemento specifico: la capacità d'imporsi come »spettacolo«. Con simili argomenti, in cui emerge senza dubbio un'intuizione lungimirante sul futuro del mondo dello spettacolo, egli finiva tuttavia per sminuire anche le esperienze teatrali più sperimentali avutesi in Italia negli anni Venti, fra cui quelle di Campanile, di Antonelli e di Pirandello, prima di tutte, oltre che le proprie, le migliori delle quali, assai innovative, risalgono al periodo compreso fra il 1919 e il $1928^{44}$. In merito a quella fase del teatro italiano Bontempelli scriveva: »C'era stata una vampata, subito spersa. ${ }^{45}$ Anche in questa critica radicale al teatro ci sembra di

\section{Cfr. AN, 28.}

$38 »[\mathrm{~N}]$ oi cerchiamo l'arte d'inventare favole e persone talmente nuove e forti, da poterle far passare attraverso mille forme e mille stili mantenendo quella forza originaria; appunto come avvenne dei miti e dei personaggi delle due ere che ci hanno preceduto.« AN, 39.

39 »Anzi la vera e suprema forma poetica, direi che non è la esaltata descrizione del proprio stato d'animo (quale è di solito intesa la poesia lirica) ma appunto la creazione di miti.« AN, 169, corsivo nel testo.

40 AN, 32 (dall'articolo apparso nel '27 col titolo »Conseils« sul III Cahier), corsivo nel testo.

41 AN, 317, corsivo nel testo

42 AN, 92, corsivo nel testo.

43 Cfr. AN, 30-32. Negli stessi anni Savinio constatava che il suo romanzo Angelica o la notte reggeva su principi tratti dal cinema.

44 Siepe a nordovest, 1919; La guardia alla luna, 1920; Nostra Dea, 1925; Minnie la candida,1928.

45 AN, 376. E di sé afferma: „Come scrittore [...] non sono mai stato nemmeno approssimativamente quello che si chiama "uomo di teatro".« AN, 373. 
scorgere un nodo problematico nella visione estetica dello scrittore poiché in nome di una tesi, per il resto convincente, come quella della basilare inadeguatezza del teatro italiano del primo Novecento a rispondere alle esigenze dell'epoca e del nuovo pubblico, egli finisce per togliere importanza anche a qualitative esperienze innovatrici.

Il Novecentismo contempla l'utopia di un'epoca radicalmente nuova, la terza agli occhi di Bontempelli dopo quelle classica e romantica, e tale progetto tocca evidentemente gli ambiti etico, esistenziale ed epistemologico ${ }^{46}$. In base a quei presupposti avrebbe dovuto imporsi, infatti, un nuovo tipo d'individuo, non più soggetto disorientato, esposto al contingente e confuso con la massa, bensì cosciente del proprio destino e dei propri compiti. Sarebbe stato urgente altresì perseguire una nuova temporalità e una nuova spazialità, capaci di fronteggiare le visioni contingenti e relative di spazio e tempo ${ }^{47}$. Solo qualche anno prima, all'altezza del lavoro sui due antiromanzi La vita intensa e La vita operosa (1919-1921) l'operazione bontempelliana volta a porre un argine al relativismo non sembrava ancora così urgente. L'esigenza era piuttosto farlo straripare, il relativismo, portarlo all'assurdo con ironia e con una volontà di denuncia ${ }^{48}$ e sperimentazione $^{49}$. Di fatto le opere stese dallo scrittore tra il primo dopoguerra e la metà degli anni Venti mostrano un'originale elaborazione di inputs, anche formali, accolti dalle avanguardie e rendono conto di esigenze condivise negli ambienti letterari europei più innovatori. Si pensi solo all'uso di manichini e burattini come attanti, a numerosi fenomeni di sorpresa, come l'uso straripante della digressione, alla pratica della metalessi fra piano testuale ed extratestuale o ancora alle situazioni surreali di molti testi, ottenute fra l'altro attraverso la decontestualizzazione di oggetti e situazioni, l'animarsi dell'oggettuale o l'oggettivarsi dell'animato. È appunto di quel periodo (siamo nella prima metà degli anni Venti) il Bontempelli che maggiormente s'avvicina ai surrealisti, per quanto il suo surrealismo non abbia certo i tratti di quello teorizzato dai francesi risultando meno straniante oltre che privo di finalità politicamente eversive ${ }^{50}$. Ed è altresì quello il Bontempelli che sta in più stretto rapporto di empatia con Pirandello, nonostante le diverse strategie abbracciate. Un cambiamento nel modo d'intendere la funzione della narrativa, col conseguente

46 Riaffiora nella considerazione dei concetti di »classico« e »romantico« la tradizione schlegeliana. Bontempelli vede quelle due epoche contraddistinte rispettivamente da uno sguardo esteriore e interiore sulla realtà. Per quanto concerne la sua tripartizione delle ere culturali, Airoldi Namer $(1975,255)$ ipotizza vi abbia pesato la filosofia di Vico, tornata in auge in quegli anni anche per merito di Croce.

47 »Il còmpito più urgente e preciso del secolo ventesimo, sarà la ricostruzione del tempo e dello spazio [...]. Quando potremo credere di nuovo in un Tempo e in uno Spazio oggettivi e assoluti, che si allontanano dall'uomo verso l'infinito [...] potremo con sicurezza affrontare il secondo còmpito, che sarà il ritrovamento dell'individuo, sicuro di sè [SIC], sicuro d'essere sè, di essere sè e non altri, sè con alcune certezze e alcune responsabilità, con le sue passioni particolari e una morale universale: e in cima a tutto ritroveremo forse un Dio, da pregare o da combattere.« AN, 17 (versione it. d'un passo di »Justification«con cui si apriva il I dei Cahiers).

48 Saccone $(1979,30)$ rileva ad esempio nel protagonista de La vita operosa (figura di letterato/reduce non integrabile nel tessuto economico-sociale) la difesa di una non omologazione, una »resistenza sottile, quasi anarchica, alla pianificazione, alle esigenze dell'apparato produttivo«.

49 Sulle strategie discorsive di quei due lavori cfr. Mascia Galateria, 1977; su La vita intensa cfr. Guglielmi 1996.

50 Fontanella $(1983,142)$ parla al proposito di un »surrealismo razionalizzato«. 
raggiustamento delle strategie retorico-discorsive, ha luogo sul finire degli anni Venti. Agisce in tale metamorfosi, il tentativo, ben registrabile in interventi di riflessione e polemica, di reagire in modo costruttivo al relativismo quale causa della malattia del proprio tempo, di cui coglieva l'origine nel peso eccessivo che era stato assegnato alla soggettività, come chiariva nell' articolo del gennaio del '28, dal titolo »Spazio e tempo«. »L'orgia metafisica dell'Io come misura unica del mondo, prima, poi come unica verità, portava fatalmente l'individuo a non credere più nemmeno a se stesso, cioè spingeva l'umanità al suicidio morale. $\ll^{51}$ Nello stesso contesto menzionava alcuni fenomeni di quell'età »decadente«, collegati all'imporsi della misura unica dell'io: dall'estetismo e impressionismo nell'arte, alla tesi che criterio di giudizio artistico sia l'intuizione individuale (con riferimento esplicito all'estetica crociana), al freudismo, tendenza volta a fare dell'umanità »una dispersione di larve vaganti dietro le pallide spinte di frammenti di immagini di sogni«, fino a un concetto deleterio di spirito democratico, inteso come »immaginaria libertà « astratta $»$ da qualunque legge superiore $\ll^{52}$. In una postilla a tale articolo, risalente al febbraio del 1929, anch'essa riportata nell'Avventura novecentista, Bontempelli riassumeva la sua diagnosi e la sfida che si apriva:

Il diavolo ha bisogno che l'uomo non creda a sé stesso e al mondo [...]. Per qualche tempo la cosa gli è riuscita per mezzo del materialismo e poi del suo luogotenente il positivismo; quando non li ha più potuti tenere in piedi, ha dovuto ricorrere al relativismo [...]. La nostra dunque è un'impresa contro il diavolo ${ }^{53}$.

La preoccupazione che motivava tale impresa era quella dell'intellettuale del tardo moderno, ben cosciente del disorientamento concettuale indotto da un pensiero basato sulla soggettività e dunque sulla relatività di ogni verità e giudizio e sull'ambivalenza dei valori. Bontempelli non faceva che condividere un disagio diffuso fra la stragrande maggioranza dei letterati del modernismo. Come rilevato da Peter Zima, la tendenza ben visibile in narratori attivi tra fine Ottocento e inizio Novecento era allora quella di cercare, ma senza troppe illusioni, un'ultima chance nella salvaguardia dei valori dell'identità, della coerenza e dell'autenticità ${ }^{54}$. Esprimendo preoccupazione rispetto a un relativismo esasperato, Bontempelli si mostra intellettuale del proprio tempo. Tuttavia il ductus utopico con cui affronta il problema, il tono apodittico che usa nell' argomentazione, la consequenzialità con cui applica le sue tesi nella produzione letteraria matura e, più in generale, la fiducia che lo porta a credere si possa mettere davvero riparo a quella tendenza di pensiero e inaugurare una temporalità svincolata dal contingente e dal soggettivo, fanno di lui un caso isolato.

51 AN, 45.

52 Ivi.

53 AN, 46, corsivo nostro.

54 Cfr. Zima, 1991. 
L'intenzione di ridare saldezza a concetti ontologici trova attuazione, nella prassi letteraria, anche in una revisione della figura del personaggio: quello con attitudine interrogante delle sue opere dei primi anni Venti, sia esso scaltro oppure al contrario candido e disorientato, tende a perdersi negli anni successivi, in concomitanza con la teorizzazione del Novecentismo e oltre, per lasciare spazio a una figura più salda che combatte una battaglia contro il tempo contingente. Paradossalmente il personaggio bontempelliano si disumanizza non nella fase in cui lo scrittore fa agire nelle sue opere anche burattini e manichini, oltre che figure calate in situazioni fuori dal mondo, ma nel momento in cui farà vivere al suo homo fictus vicende da cronaca, attribuendogli tuttavia i tratti di un'umanità superiore ${ }^{55}$. Tale trasformazione è esplicitata anche in alcune note critiche, confluite poi nell'Avventura novecentista, in cui si esprime appunto la volontà di contrapporre l'eroe alla figura d'inetto dei romanzi francesi tedeschi e austriaci. Riferendosi nella fattispecie alla narrativa di Céline, Bontempelli affermava: »L'uomo di debole volontà, preda dei suoi più facili istinti, incapace di ogni risoluzione, antivirile, è il tipo prediletto messo di moda da questi detriti del più basso romanticismo europeo. $\aleph^{56}$ In quel contesto (si tratta di una lettera del 1934 a Bompiani) scriveva in termini di obiezione: „L'arte narrativa che si vuole imporre all'Italia è un'arte di disfacimento. Noi sentiamo il romanzo come poema, il personaggio come eroe. Eroe del bene o del male. $\ll^{57}$ A condurlo a quella svolta poetologica non c'era solo il bisogno di riproporre in ambito narrativo un personaggio che rappresentasse un soggetto integro e orientato, ma vi giocava un ruolo centrale anche una preoccupazione etica. E però nel perseguire un simile intento nella propria attività di narratore, egli avrebbe finito per scrivere dei testi meno carichi di quel senso dell'ambiguo riscontrabile nelle sue opere dei primi anni Venti risultando meno convincente.

Volendo trarre un breve bilancio, il progetto ideologico-culturale novecentista di superamento del relativismo appare, specie se letto a posteriori tenendo presente gli sviluppi del pensiero e delle lettere dagli anni Trenta in poi in Occidente, una lotta persa in partenza. Verrebbe da dire che Bontempelli aveva sottovalutato la forza del »diavolo«. Il relativismo e con esso la crisi d'identità e la perdita di salde categorie ontologiche non sarebbero stati fenomeni arrestabili, tali da essere contrastati. Restano tuttavia innegabili la straordinaria lucidità con cui egli individuò l'essenza della modernità novecentesca e il convinto e coerente impegno adoperato nella ricerca di una poiesis capace di corrisponderle.

55 Luciana, nel romanzo Il figlio di due madri (1929), o Adria, in Vita e morte di Adria e dei suoi due figli (1930), o ancora le figure delle nipoti, in Gente del tempo (1937), adempiono in modo conseguente il destino che incombe su di loro.

56 AN, 265.

57 »Quella gente [coloro che vorrebbero imporre "un romanzo di disfacimento"] vede il romanzo sotto la sola specie di trattato di storia naturale, e non sa trattarvi l'uomo che come fosse un coleottero, i costumi degli uomini come la vita d'un formicaio. Non voglion ch'esistano "persone" [...] c'è tra i romanzieri una morbosa corsa al materiale umano più basso.«Ivi. 


\section{BIBLIOGRAFIA}

(abbreviazione) AN = BONTEMPELLI, Massimo (1938) L'avventura novecentista. Firenze: Vallecchi.

ALVARO Corrado, BONTEMPELLI Massiomo, FRANK Nino (1985) Lettere a "900", a cura di Marinella Mascia Galateria, Roma.

BONTEMPELLI, Massimo (1946) Dignità dell'uomo. Milano: Bompiani.

BONTEMPELLI, Massimo (1978)5 Opere scelte, a cura di Luigi Baldacci. Milano: A. Mondadori.

BONTEMPELLI, Massimo (2006) Realismo magico e altri scritti sull'arte, a cura di Elena Pontiggia. Milano: Abscondita.

AIROLDI NAMER, Flavia (1975) Gli scritti teorici di Massimo Bontempelli nei "Cahiers du 900» e la ricostruzione mitica della realtà. (Studi novecenteschi IV, 12). 248-269.

BRIOSCHI, Franco (2002) Critica della ragion poetica. Torino: Bollati Boringhieri.

BUONANNO, Errico (2003) Il novecento immaginario di massimo Bontempelli. (Studi novecenteschi, 30, 66), 239-262.

CONTINI, Gianfranco (1972) Altri esercizi. Torino: G. Einaudi.

FALQUI, Enrico (1953) Il Futurismo, Il Novecentismo. Torino: Edizioni Radio italiana.

FALQUI, Enrico (1967) Introduzione a: Giuseppe De Robertis, Scritti vociani, a cura di Enrico Falqui. Firenze: F. Le Monnier.

FONTANELLA, Luigi (1983) Il surrealismo italiano, Roma: Bulzoni.

FONTANELLA, Luigi (1992) Bontempelli tra mito e metafisica, in Massimo Bontempelli, scrittore e intellettuale. Atti del Convegno (Trento, 18-20 aprile 1991), a cura di Corrado Donati e Fulvia Airoldi Namer, Roma, 99-112.

FONTANELLA, Luigi (1997) Storia di Bontempelli, Ravenna: Longo.

GUGLIELMI, Guido (1996) Un romanzo manifesto, in: Id., La prosa italiana del Novecento. Umorismo, metafisica, grottesco, Torino: Einaudi. 198-210.

MASCIA GALATERIA, Marinella (1977) Tattica della sorpresa e romanzo comico di Massimo Bontempelli. Roma: Bulzoni.

MILANKO Sandra (2014) Bontempelli, l'avanguardia, il pubblico: dal futurismo alla pittura metafisica. Tesi dottorale, Università degli studi di Trieste; consultabile online: www.openstarts.units.it/xmlui/bitstream/handle/10077/10152/tesi\%20sandra\%20milanko.pdf?sequence=1 (1.11.2014).

ORTEGA Y GASSET, José (1997) La deshumanización del arte y otros ensayos de estética. Madrid: Revista de Occidente.

PISCOPO, Ugo (2001) Massimo Bontempelli. Per una modernità dalle pareti lisce. Napoli: Edizioni scientifiche italiane. 
SACCONE, Antonio (1979) Massimo Bontempelli, Il mito del '900. Napoli: Liguori. ZIMA, Peter (2001) Das literarische Subjekt. Zwischen Spätmoderne und Postmoderne. Tübingen-Basel: Francke.

\section{SOMMARIO}

Il Novecentismo: »Un'impresa contro il diavolo«. Note sul progetto culturale ed estetico bontempelliano

Nel presente contributo, sulla scorta degli interventi teorici e polemici raccolti ne L'avventura novecentista (1938) si ripercorrono le posizioni di Bontempelli legate al progetto di rinnovamento culturale (il Novecentismo) di cui questo intellettuale fu promotore negli anni Venti. Vi si segnalano le battaglie difese e la lungimiranza delle vedute, ma anche i nodi problematici o piuttosto le sfide che quel progetto lanciava. E qui rientrano l'utopica battaglia contro il relativismo, la difesa dell'autonomia dell'arte assieme alla contemporanea richiesta di una sua apertura alle esigenze del proprio tempo, la ricerca di conciliare un fare estetico orientato alla rottura di norme e attese con un concetto di arte e letteratura popolari.

Parole chiave: Novecentismo, realismo magico, rinnovamento culturale, autonomia dell'arte, superamento del relativismo.

\section{ABSTRACT \\ Novecentism: Battle Against the Devil. Notes about Bontempelli's Cultural and Aesthetic Project.}

This article, with reference to theoretical and polemical essays collected in L'Avventura novecentista (1938), retraces Bontempelli's perspective on the project of cultural renewal (the Novecentism), which he himself promoted in the 1920's. This article presents not only the battles he sustained and his farsightedness, but also the problems or rather the challenges of this project. Along these challenges came the utopian battle against relativism, the tendency to defend the autonomy of art and the contemporary requirement that this autonomy be open to the necessities of that time, as well the attempt to conciliate an artistic work, inclined to break the norms and expectations, with a concept of popular literature and art.

Keywords: Modernism, magic realism, cultural renewal, autonomy of art, overcoming of relativism. 


\section{POVZETEK}

\section{Novecentizem: Boj z mlini na veter. Nekaj beležk o Bontempellijevem kulturnem in estet- skem projektu}

Pričujoči prispevek s pomočjo teoretskih in polemičnih spisov, zbranih v delu L'avventura novecentista (1938), osvetljuje Bontempellijevo stališče do projekta kulturne prenove, ki jo je v dvajsetih letih minulega stoletja sam spodbujal. V članku so predstavljeni njegovi kulturni boji in daljnovidnost pogledov, pa tudi težave - ali bolje rečeno izzivi, ki jih je projekt sprožil.

Sem sodijo utopični boj proti relativizmu, težnja po zaščiti avtonomije umetnosti skupaj s sočasno zahtevo, da se ta odpre zahtevam in izzivom tistega časa, prav tako pa tudi poskus združitve estetskega dela, usmerjenega h kršenju norm in pričakovanj, s konceptom ljudske umetnosti in književnosti.

Ključne besede: Novecentizem, magični realizem, kulturna prenova, avtonomija umetnosti, boj proti relativizmu. 